\title{
Performance analysis of direct-sequence spread-spectrum underwater acoustic communications based on at-sea data
}

\author{
Pengyu Du*, Lujun Wang, Hongtao Zhang, and Zhe Xie \\ Science and Technology on Sonar Laboratory, Hangzhou Applied Acoustic Research Institute, Hangzhou, 310023
}

\begin{abstract}
Direct sequence spread spectrum (DSSS) signal uses phase coherent signals where the information symbols are multiplied with a code sequence, commonly known as chips. The signals are processed at the receiver using the code sequence as a matched filter to extract the information symbols. Taking advantage of the spread processing gain derived from the matched filter, communications can be carried out at low signal levels, which is the preferred method for high quality underwater acoustic (UWA) communication and remote UWA communication. However, in practical applications, DSSS UWA communication will face lots of interferences including multipath interference, Doppler compression interference and phase fluctuation interference, which will seriously affect the performance of DSSS. This paper first analyzes the interferences that DSSS faces in practical applications based on multiple actual received data collected in different water areas. By calculating the gain of the matched filter loss, one can find that influence of phase fluctuation interference has the most serious impact on DSSS. Secondly, the corresponding receiver processing algorithms are given for these interferences, including the differential energy detector and Doppler estimation method. The performance of differential energy detector under the above interferences is analyzed, which shows that the differential energy detector is insensitive to slow phase fluctuation interference and multipath interference and the differential energy detector combined with Doppler compensation can work well based on moving at-sea data. Finally, the parameter design of DSSS system in practical application is discussed and parameter design of DSSS UWA communication system should comprehensively consider the Doppler compression interference and phase fluctuation interference.
\end{abstract}

\section{Introduction}

As a means of underwater information transmission, underwater acoustic communication not only has a strong military application background, but also is indispensable in the civilian field. The underwater acoustic (UWA) channel is a time-frequency, spacevarying channel with limited bandwidth and severe multipath interference[1]. The complexity of UWA channels makes high-quality UWA communication a challenge.

Direct sequence spread spectrum (DSSS) communication has good anti-interference and multipath resistance and can work under low signal-to-noise ratio conditions, which is the preferred communication method for high-quality UWA communication[2-4]. In DSSS UWA communication system, the carrier phase fluctuation will seriously affect the spread spectrum gain resulting in system performance degradation. Phaselocked loop can track the phase of the locked carrier in real time, which ensures that the spread spectrum gain is not affected by the carrier phase fluctuation[5]. However, under low SNR conditions, the phase noise in the phaselocked loop will seriously affect its performance. In fact, carrier phase fluctuation interference is the primary problem faced by DSSS UWA communication in practical applications. Carrier phase fluctuation interference will directly affect the symbol decision at the receiving end and fast carrier phase fluctuation interference will cause the receiver to lose spread spectrum gain, which in turn will cause decoding failure.

DSSS communication uses the quasi-orthogonality of pseudo-random sequences to suppress interference. However, the multi-path extended interference of the UWA channel will seriously affect the orthogonal characteristics of the pseudo-random sequence, thereby reducing the decoding performance. In response to this problem, related technologies such as DFE and PPC have been proposed[6-7]. However, DFE technology requires a high input signal to noise ratio and PPC technology requires real-time updates to the estimated channel, in practical applications, both of which will be subject to certain restrictions in practical applications. In addition, since the DSSS signal is generally long, it will be more affected by Doppler compression/expansion, which will place higher requirements on the code side synchronization at the receiving end.

In this paper, the actual collected data will be used to analyze the factors affecting the performance of DSSS communication, so as to clarify the extent of the influence of each main interference factor on DSSS communication. A DSSS receiver algorithm was

\footnotetext{
* Corresponding author: heaven663@163.com
} 
proposed in the previous research work[8], and this paper will verify the applicability of the method under various major interference factors. At the same time, a Doppler estimation method is given for the Doppler Effect. Finally, for practical applications, this paper will discuss and suggest the parameter design of the direct expansion underwater acoustic communication system. The structure of this paper is as follows: section 2 analyses the interference faced by direct water-acoustic communication. Section 3 introduces the principle of differential energy detector (DED) and analyzes its performance. At the same time, a Doppler estimation method is given. Section 4 discusses the selection of system parameters in the practical application of DSSS communication.

\section{LHEX12 and QDEX17 Data}

This paper will analyze the DSSS signal data collected in two different waters, which are from the LHEX12 (LianHua Lake Experiment) in 2012 and the QDEX17 (QianDao Lake Experiment) in 2017.

The LHEX12 data bandwidth is $5 \sim 7 \mathrm{kHz}$, and the spread spectrum sequence uses 7 th order $\mathrm{m}$ sequence. Fig. 1 shows the results of the measured UWA channel in LHEX12. The multi-path structure of the UWA channel in this water area is complicated and the multi-path expansion is serious. It can be seen from Fig.1(a) that the maximum multi-path extension is nearly $60 \mathrm{~ms}$. Fig. 1(b) shows the time-dependent characteristics of the measured channel. It can be seen that the channel undergoes a slow time-varying during the observation time. Fig.1(c) shows the correlation characteristics of the channel in the duration of adjacent spread spectrum symbols $(\sim 63 \mathrm{~ms})$. It can be seen that the underwater acoustic channel can be considered to be constant during the observation time of $126 \mathrm{~ms}$. Since the transceiver node is relatively stationary and the lake surface is relatively quiet, the carrier phase fluctuation interference in the ExLH12 data is small. However, due to the large multi-path spread interference, the gain of the matched filter output decreases significantly. Fig. 2 shows the DSSS signal matching filter output under the condition of receiving signal-to-noise ratio (SNR) of $20 \mathrm{~dB}$. It can be seen that the peak of the matching output is not obvious due to the influence of multi-path expansion.
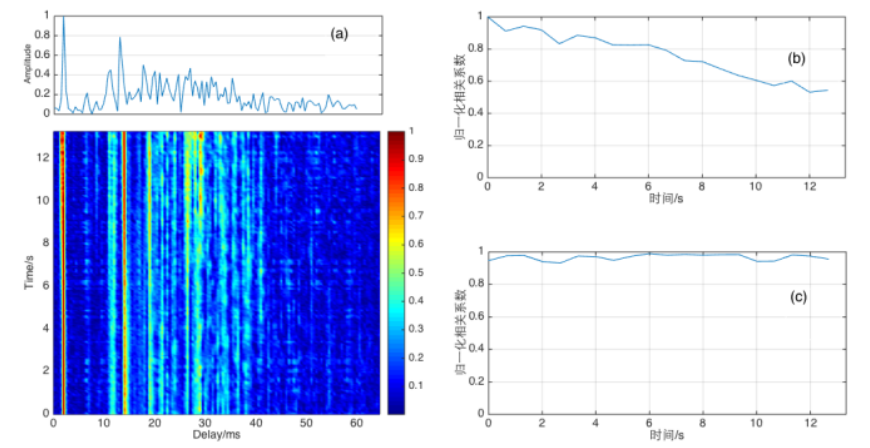

Fig. 1. ExLH12 measured UWA channel.

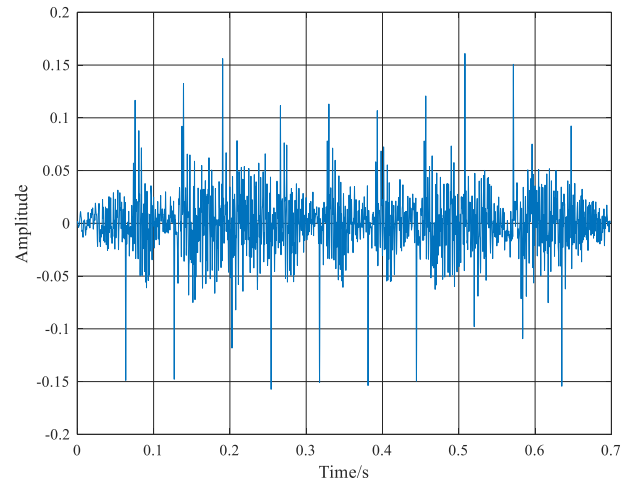

Fig. 2. Matched filter output.

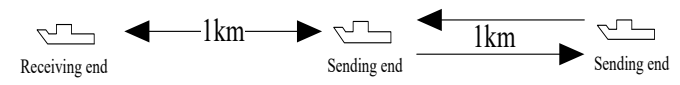

Fig. 3. EXQD17 test layout.

The QDEX17 data bandwidth is $1.5 \sim 1.8 \mathrm{kHz}$, and the spread spectrum sequence uses 7 th order $\mathrm{m}$ sequence. The main content of EXQD17 is low-frequency remote mobile underwater acoustic communication, so the transceiver node has 4 6 knots relative motion, and the transmission power is strictly controlled to simulate the remote communication conditions. Fig. 3 shows the schematic diagram of the test process. The receiving end is fixed and the transmitting end moves back and forth 1 $\mathrm{km}$ away from the receiving end. The transmitting end continuously adjusts the transmitting power during the motion, and the receiving end performs real-time decoding and the decoded information is fed back to the transmitting end through the radio. Since the received signal is affected by the Doppler effect, the receiving end will lose the matched filter processing gain. Fig.4 shows the comparison of the matched filter output before and after Doppler compensation. It can be seen that the Doppler effect causes fast carrier phase fluctuation interference in the received signal, so that the matched filter cannot output the correlation peak, as shown by the blue line in Fig.4. After the Doppler compensation, the matched filter outputs a significant correlation peak, as shown by the red line in Fig. 4.

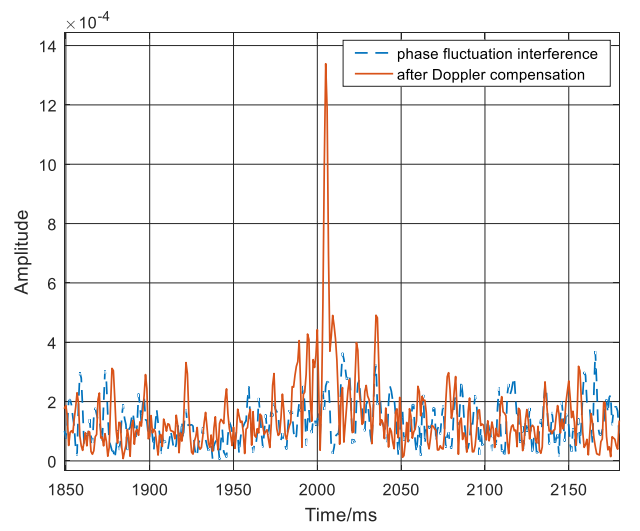

Fig. 4. Matching output comparison before and after Doppler compensation.

It can be seen from the actually received DSSS signal that the main factor affecting DSSS communication performance is Doppler Effect. It causes the received 
signal to be interfered by fast carrier phase fluctuation, which causes the direct-spreading receiver matching filter to lose the matching processing gain. The multipath extended interference is another factor that affects the performance of DSSS communication. Multi-path extended interference will also destroy the quasiorthogonal characteristics of the pseudo-random sequence, but its influence degree is significantly lower than that of fast carrier phase hopping interference. In this paper, a DS receiver algorithm and Doppler estimation method will be given in the next section, and the proposed method will be analyzed and verified by using the data collected in the above two waters.

\section{DSSS receiver and performance analysis}

The receiving end of the DSSS system uses a differential energy detector (DED) for decoding, and its schematic diagram is shown in Fig.5. After the coarse synchronization, the received signal enters the DED in units of two spread symbol periods, and performs correlation operations with the two sets of locally constructed spread spectrum sequences. The DED finally decodes by comparing the energy outputs of the two correlators. At the same time, DED feeds back the time corresponding to the maximum value of the energy output, and completes the synchronous updating of the coded bits of the spread spectrum, which can effectively avoid the Doppler compression/expansion effect. The following uses the formula to further explain the DED.

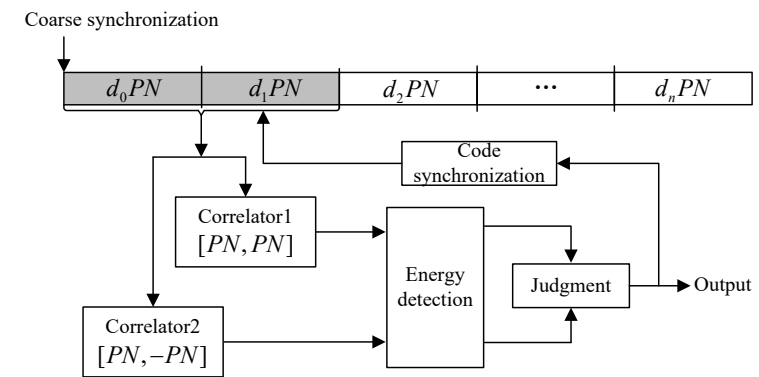

Fig. 5. Differential energy detector schematic.

The original information sequence is differentially encoded and then transmitted after being spread and carrier modulated. At the receiving end, the differential energy detector first constructs a pair of combined sequences using local reference spreading sequences

$$
P_{+}=[c, c], \quad P_{-}=[c,-c]
$$

The received DSSS baseband signal enters the DED in units of two spread symbol periods. Let the baseband signal entering the DED each time be $r_{n}$, one can get:

$$
r_{n}=\left[d_{n-1} c e^{j \varphi_{n-1}}, d_{n} c e^{j \varphi_{n}}\right]+\Gamma_{n}
$$

The result of correlator 1 and correlator 2 outputs of the differential energy detector is

$$
\begin{aligned}
& E_{1}=\left|\left\{\left\langle P_{+} \cdot r\right\rangle\right\}_{T}^{3 T}\right|^{2}=\left|\left(d_{n-1} e^{j \varphi_{n-1}}+d_{n} e^{j \varphi_{n}}\right)\right|^{2} \rho^{2}+\Delta_{1} \\
& E_{2}=\mid\left\{\left.\left\langle P_{-} \cdot r>\right\}_{T}^{3 T}\right|^{2}=\left|\left(d_{n-1} e^{j \varphi_{n-1}}-d_{n} e^{j \varphi_{n}}\right)\right|^{2} \rho^{2}+\Delta_{2}\right.
\end{aligned}
$$

where $|\cdot|$ and $\langle\cdot\rangle$ is modulo operation and crosscorrelation operation respectively and $\rho$ is the spread spectrum sequence autocorrelation function. $\{\cdot\}_{T}^{3 T}$ indicates that the output signal is intercepted from time $T$ to time $3 T$, and $\Delta_{1}$ and $\Delta_{2}$ are despread noise components, which are negligible for small quantities. Since $\varphi_{n-1} \approx \varphi_{n}$, we can get

$$
\begin{aligned}
& E_{1}=\left|e^{j \varphi_{n}}\right|^{2}\left|d_{n-1}+d_{n}\right|^{2} \rho^{2}+\Delta_{1} \\
& E_{2}=\left|e^{j \varphi_{n}}\right|^{2}\left|d_{n-1}-d_{n}\right|^{2} \rho^{2}+\Delta_{2}
\end{aligned}
$$

Therefore, if $\max \left\{E_{1}\right\}>\max \left\{E_{2}\right\}$, then $d_{n-1} d_{n}=a_{n}=1$; otherwise $d_{n-1} d_{n}=a_{n}=-1$. Decoding is accomplished by comparing the correlator output energy matching results. At this time, the output results are all real numbers, so the DED will not be affected by carrier phase fluctuation interference when the carrier phase changes slowly.

When considering the influence of the underwater acoustic channel, equation (4) will become:

$$
\begin{aligned}
& E_{1}=\left|e^{j \varphi_{n}}\right|^{2}\left|d_{n}+d_{n+1}\right|^{2}\left(\rho \otimes h_{n}\right)^{2}+\Delta_{1} \\
& E_{2}=\left|e^{j \varphi_{n}}\right|^{2}\left|d_{n}-d_{n+1}\right|^{2}\left(\rho \otimes h_{n}\right)^{2}+\Delta_{2}
\end{aligned}
$$

Since the DED algorithm compares the energy output results of two correlators, it can be known that when the UWA channel multipath expansion is less than the spread symbol period, the multipath extended component of the UWA channel will become a beneficial contribution of energy. Fig. 6 shows the DED output of the QDEX17 data. It can be seen that the DED output verifies the analysis result of equation (5).

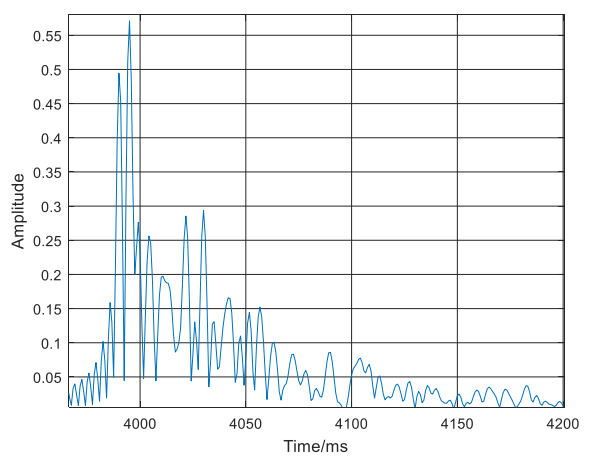

(a)

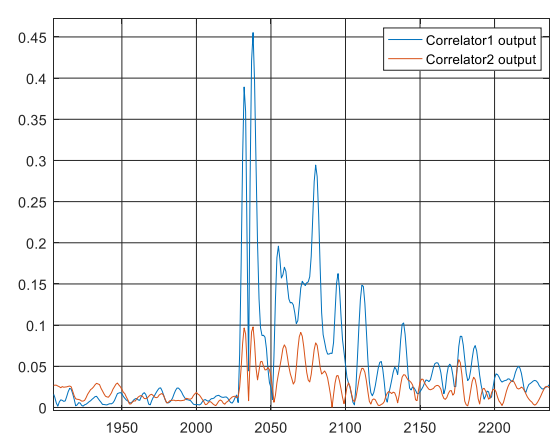

(b)

Fig. 6. The DED decoding output of QDEX17 data. (a) Measured UWA channel; (b) DED output. 
The application of the DED is based on the fact that the carrier phase remains stable for the duration of the adjacent spread symbol, but this condition will not be met when there is significant relative motion. Therefore, in mobile UWA communication, the DED must first perform Doppler estimation and compensation before decoding. As shown in Fig.7, this paper estimates the Doppler of the received DSSS signal by locally constructing a multi-channel received signal Doppler replica. Fig.8 shows the QDEX17 received data Doppler estimation result.

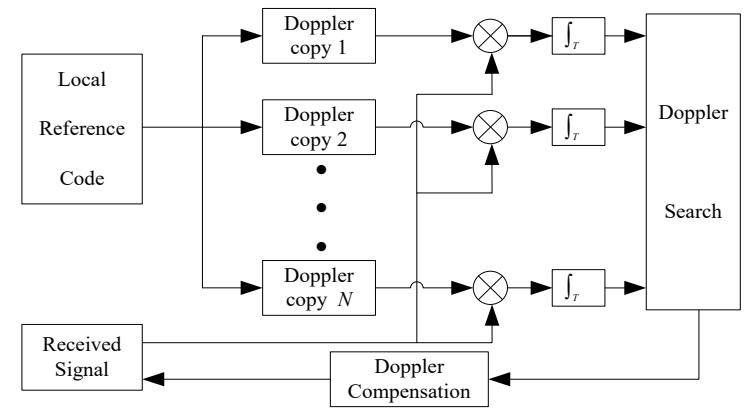

Fig. 7. Schematic diagram of Doppler estimation method

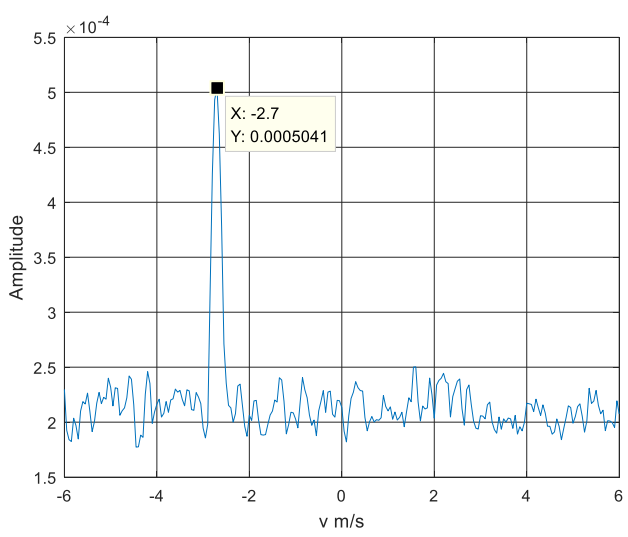

Fig. 8. Doppler estimation results.

\section{Discussion on DSSS communication in practical application}

\subsection{Doppler Effect}

The core of DSSS UWA communication is to obtain matched filter processing gain and the fast carrier phase fluctuation interference caused by Doppler Effect is the most important factor affecting the matched filter processing gain. Let the transmission signal be

$$
s(t)=m(t) \cos \left(\omega_{c} t\right)
$$

where $m(t)$ is a pseudo-random sequence and $\omega_{c}$ is the carrier center frequency. If the transmitting and receiving end have a relative motion of speed $v$, the received signal is

$$
r(t)=m^{\prime}(t) \cos \left(\omega_{c} t+\delta \omega_{c} t\right)
$$

where $\delta=v / c$ is the Doppler coefficient and $m^{\prime}(t)=m[(1+\delta) t]$ is the signal envelope after Doppler compression. It can be seen that the influence of Doppler on the spread spectrum signal is reflected in two aspects: a) compression or broadening of the spread spectrum sequence; b) significant carrier phase fluctuation interference of the received signal. It can be known from equation (7) that the carrier phase fluctuation interference is not only related to the relative motion velocity $v$, but also related to the carrier center frequency, that is, the higher the center frequency under the same conditions, the more severe the system is interfered by the carrier phase fluctuation. Under the same conditions, Fig.9 shows the matched filter output results of the spread spectrum signals with carrier center frequency $6 \mathrm{kHz}$ and $18 \mathrm{kHz}$, respectively. The relative motion speed of the transceiver node is $0.5 \mathrm{~m} / \mathrm{s}$, and Doppler compensation is not performed before the matched filter processing. It can be seen that under the influence of carrier phase fluctuation, the normalized correlation peaks of the two spread spectrum signal matched filter outputs are all decreased, and the higher the carrier center frequency, the faster the normalized correlation peak falls.

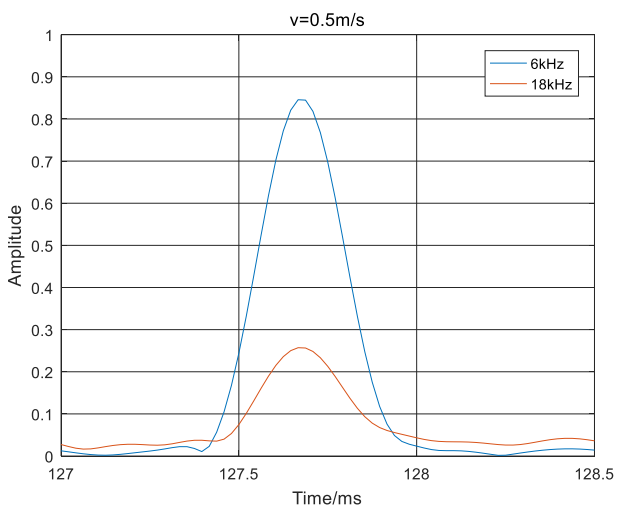

Fig. 9. Matched filter output at different center frequencies with Doppler Effect

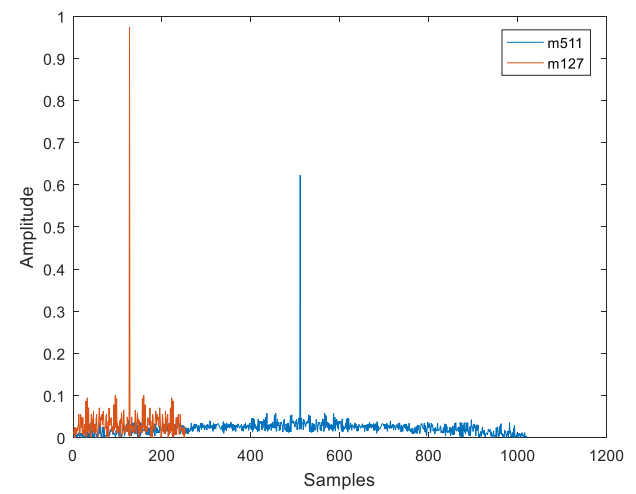

Fig. 10. Matched filter output under different spreading symbol periods

In addition, the carrier phase fluctuation interference is a relative quantity, and the longer the duration of the spreading symbol, the more severe the interference caused by the carrier phase fluctuation. Fig. 10 shows the simulation results of the spread spectrum signal matching filter output using the 7 th order $\mathrm{m}$ sequence and the 9th order $m$ sequence under the same conditions. The relative motion speed of the transceiver node is $0.5 \mathrm{~m} / \mathrm{s}$, and Doppler compensation is not performed before the matched filter processing. It can be seen that the spread spectrum signal with the 9th order sequence has a longer spread spectrum period, and the normalized 
correlation peak of the matched filter output falls faster under the same carrier phase interference.

The above analysis results show that in practical applications, the parameters of the DSSS system should be selected at a lower center frequency if the conditions are met. At the same time, although the longer the spreading sequence theoretically obtains higher processing gain, the output loss of the matched filtering is more serious due to the influence of carrier phase fluctuation interference and Doppler compression extended interference.

\subsection{Multipath interference of UWA channel}

Multipath interference will destroy the orthogonal characteristics of the spread spectrum sequence and reduce the gain of the matched filter processing. At present, the commonly used method for suppressing multipath interference is to use the TRM technique. However, the premise of applying TRM is to estimate the UWA channel. Since the DSSS signal usually works at a low SNR, it brings certain difficulties to channel estimation. One solution is to add a detection signal with a high matching gain before the DSSS signal. Fig.11 shows the output of the DED before and after the TRM processing of the LHEX12 data. It can be seen that the TRM processing significantly improves the system decoding performance.

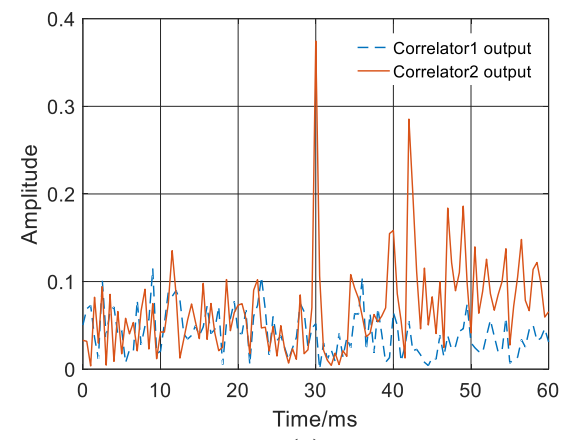

(a)

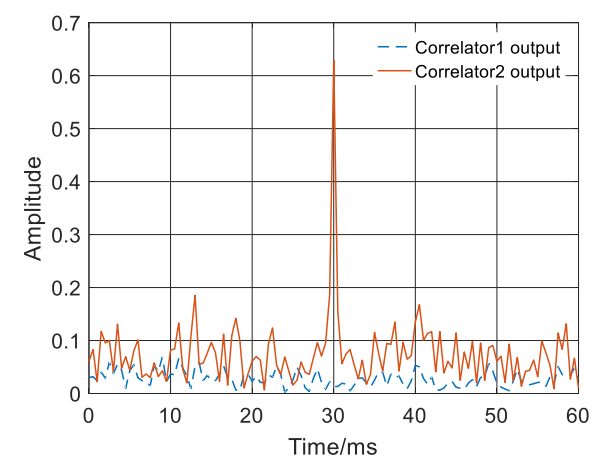

(b)

Fig. 11. DED output before and after TRM processing. (a) without TRM; (b) with TRM

For QDEX17 data, TRM is no longer applicable. This is because the receiving spread spectrum symbol has a long period of duration, and there is significant relative motion between the transmitting and receiving parties, so the multipath structure of the UWA channel will rapidly change. The TRM processing with the estimated channel does not achieve the desired effect. Therefore, for multipath interference, the DSSS signal should be designed to ensure that the signal duration is short enough, or to insert multiple synchronization signals in the DSSS signal to estimate and update the channel in real time. For low-frequency long-range spread spectrum communication signals, the channel multipath interference is much smaller than the spreadspectrum symbol duration. At this time, the multipath interference suppression can be realized by using the DED's own anti-multipath capability.

\subsection{Command communication}

Although the direct-expansion underwater acoustic communication has high reliability, its communication rate is too low, so it is mostly used to transmit command information. A necessary condition for command communication is that the receiving end must know whether the decoded information is correct, otherwise the opposite instruction due to the decoding error may have fatal consequences. Therefore, the CRC check code is added to the original instruction information, and the $\mathrm{CRC}$ check by the receiving end after decoding is a method for ensuring that the command information is not false positive. The CRC check code constitutes a CRC sequence by adding $\mathrm{N}$ bits information after the original information sequence, and the check false positive probability is $1 / 2^{\mathrm{N}}$. Here again, there will be a problem: if $\mathrm{N}$ is chosen to be large, the redundancy of the system is seriously increased, and the communication efficiency is significantly reduced; if the selection of $\mathrm{N}$ is too small, the risk of false alarms is increased. This paper solves this problem by using the DED to have low synchronization requirements. Fig.12 shows the information flow of the command communication receiving end. The received signal is first decoded by the $\mathrm{DED}$, and then the decoded information is CRC checked. If the verification is successful, the received signal is delayed by $\mathrm{t}$ and then decoded again by the DED, and perform CRC secondary verification. If the verification is successful, the command information is output. Two CRC checks will significantly reduce the probability of false positives and improve the reliability of the command communication system.

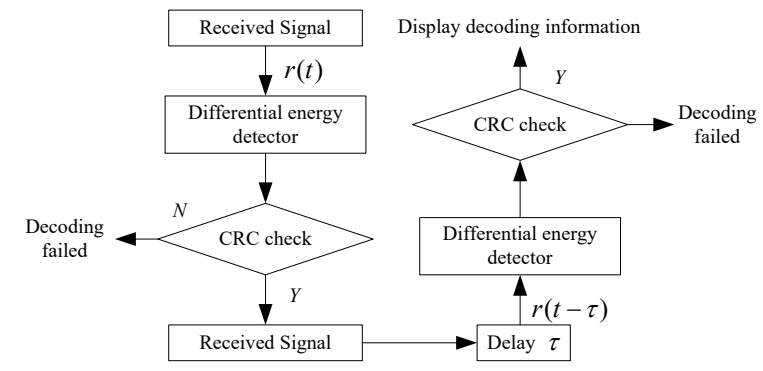

Fig. 12. Command communication receiving end information flow

\section{Conclusions}

For LHEX12 data, multipath interference is more serious and the carrier phase fluctuation interference is 
smaller and for QDEX17 data, carrier phase fluctuation interference is more serious and the multipath interference is relatively small. The receiving end of the DSSS system mainly uses a DED for decoding. The processing results of the two test data by DED show that the DED has strong anti-multipath interference capability and certain anti-carrier phase fluctuation interference capability. When there is significant Doppler Effect in the DSSS system, the receiver must perform Doppler estimation and compensation. In practical applications, Doppler cannot be accurately estimated for various reasons. Therefore, the processing result of Doppler search estimation and compensation method given in this paper is to convert large Doppler Effect into smaller Doppler Effect and the DED is then used to suppress residual Doppler Effect. At the end of this paper, the parameter design of the DSSS system in practical application is briefly discussed and analyzed. The command communication scheme based on CRC secondary verification is given, which can effectively improve the reliability of the communication system.

This work was supported by the National Natural Science Foundation of China (Grant Nos. 61701449, 61701450) and by the Innovation Special Zone of National Defense Science and Technology.

\section{References}

1. D. B. Kilfoyle, A. B. Baggeroer, IEEE J. Ocean. Eng. 25, 1 (2000)

2. G. Zhang, H. Dong, Applied Acoustics 73,9 (2012)

3. F. Blackmon, E. M. Sozer, M. Stojanovic, J. Proakis, IEEE Oceans Conf. 1, (2002).

4. T. C. Yang, W. B. Yang, J. Acoust. Soc. Am., 2008, 123, 2(2008).

5. M. Stojanovic, J. A. Catipovic, J. G. Proakis, IEEE J. Oceanic Eng. 19, 1 (1994).

6. M. Stojanovic, L. Freitag, IEEE Oceans Conf. 1, 8(2004).

7. P. Hursky, M. B. Porter, M. Siderius, et al. J. Acoust. Soc. Am. 120, 1(2006).

8. J. Yin, P. Du, X. Zhang, G. Zhu, Acta Phys. Sin. 65, 4(2016). 